\title{
Correlated Magnetoexcitons in Semiconductor Quantum Dots at Finite Temperature
}

\author{
D.J. Dean ${ }^{\dagger}$, M.R. Strayer ${ }^{\dagger}$, and J.C. Wells* \\ ${ }^{\dagger}$ Physics Division and *Computer Science and Mathematics Division, \\ Oak Ridge National Laboratory, Oak Ridge, TN 37831
}

\begin{abstract}
We describe computational methods for the theoretical study of explicit correlations beyond the mean field in excitons confined in semiconductor quantum dots in terms of the Auxiliary-Field Monte Carlo (AFMC) method [1]. Using AFMC, the many-body problem is formulated as a Feynman path integral at finite temperatures and evaluated to numerical precision. This approach is ideally suited for implementation on highperformance parallel computers. Our strategy is to generate a set of mean-field states via the Hartree-Fock method for use as a basis for the AFMC calculations. We present preliminary results.
\end{abstract}

\section{INTRODUCTION}

Revolutionary nanofabrication techniques, such as atomic-layer epitaxy, advanced lithography, and electrochemical and molecular self-assembly methods, have opened the way to fabricating quantum dots, arrays of quantum dots, and other structures that may provide the basic building blocks for future nano-scale electro-optic devices [2]. Recent experimental studies of optical emission from semiconductor quantum dots have demonstrated the importance of many-body effects, such as correlation and exchange, in exciton dynamics [3]. Intensive basic research is ongoing to accurately simulate the complex properties of current and future nanostructure devices using high-performance computers (e.g., see [4]). While significant progress has been made in the simulation of the relevant independent-particle phenomena, much important work remains in understanding and simulating the collective and correlated many-body phenomena observed in nanoscale devices at finite temperature [5]. Theoretical research has focused largely on mean-field or one-body aspects of the phenomena at zero temperature. Many-body effects, such as exciton-exciton interactions, are still lacking a complete theoretical understanding and a numerical implementation.

The focus of this paper is the theoretical description and exact, fully correlated numerical simulation of many-electron correlations in two-dimensional semiconductor quantum dots at finite temperature and exposed to strong magnetic fields. We will approach this many-body problem via the Auxiliary-Field Monte-Carlo (AFMC) method [6], which has been known since the 1950s [7], but without wide application to fermion systems because of difficulties in numerical implementations known as the "fermion-sign" problem. $\mathrm{Nu}$ clear physicists have pioneered the modern development of AFMC for the shell-model 
problem [1], obtaining practical solutions to the associated numerical issues. With this important work as inspiration, AFMC has been applied in condensed-matter systems [8] and atomic and molecular physics [9]. In those cases for which practical solutions to the sign problem have been found, the results have been quite revolutionary for the theoretical description of the phenomena. No one, to our knowledge, has tried to apply the AFMC method to quantum nanostructures. This new effort will provide a numerically exact treatment of the quantum many-body problem in nanostructures at finite temperature.

In describing the ground-state and low-lying (intraband) excitations of the N-electron semiconductor nanostructures, it is often sufficient to restrict consideration to the conduction band using the effective-mass approximation [2]. Denoting the operators of creation and annihilation of an electron in a single-particle state $|\alpha\rangle$ by $c_{\alpha}^{\dagger}$ and $c_{\alpha}$, respectively, the Hamiltonian of the N-electron system can be written in the occupation-number representation in the form

$$
\hat{H}_{e e}=\sum_{\alpha} E_{\alpha}^{e} \hat{c}_{\alpha}^{\dagger} \hat{c}_{\alpha}+\frac{1}{2} \sum_{\alpha \beta \gamma \delta}\left\langle\alpha \beta\left|V_{e e}\right| \gamma \delta\right\rangle \hat{c}_{\alpha}^{\dagger} \hat{c}_{\beta}^{\dagger} \hat{c}_{\delta} \hat{c}_{\gamma},
$$

with single-particle energies $E_{\alpha}^{e}$ and matrix elements of the Coulomb interaction between electrons $\left\langle\alpha \beta\left|V_{e e}\right| \gamma \delta\right\rangle$. In the following, we will consider nanostructures exposed to strong magnetic fields, including the Zeeman spin splitting. For present purposes, we restrict ourselves to harmonic-confining potentials.

\section{AFMC METHOD}

To calculate expectation values in AFMC, we make use of the Euclidian-time manybody propagator $\hat{U}=\exp \left(-\beta \hat{H}_{e e}\right)$, where $\beta \equiv T^{-1}$ is interpreted as the inverse of the temperature. For example, we calculate the expectation value of some observable $\hat{\Omega}$ in a canonical ensemble, with fixed particle number $N$, as

$$
\langle\hat{\Omega}\rangle=\frac{\operatorname{Tr}\left[\hat{P}_{N} \hat{U} \hat{\Omega}\right]}{\operatorname{Tr}\left[\hat{P}_{N} \hat{U}\right]}=\frac{\operatorname{Tr}_{N}[\hat{U} \hat{\Omega}]}{\operatorname{Tr}_{N}[\hat{U}]}
$$

where the many-body trace is defined as $\operatorname{Tr} \hat{X} \equiv \sum_{i}\langle i|\hat{X}| i\rangle$ and the sum is over all manybody states of the system. If $\hat{N}$ is the number operator and $\hat{P}_{N}=\delta(N-\hat{N})$ is the projector onto states with $N$ electrons, the canonical ensemble is defined by $\operatorname{Tr}_{N} \hat{X} \equiv$ $\sum_{i}\left\langle i\left|\hat{P}_{N} \hat{X}\right| i\right\rangle$.

Beyond such static properties, this approach allows one to obtain information about the dynamical response of the system. For operators $\hat{\Omega}^{\dagger}$ and $\hat{\Omega}$, the response function $R_{\Omega}(\tau)$ in the canonical ensemble is defined as

$$
R_{\Omega}(\tau) \equiv \frac{\operatorname{Tr}_{N} e^{-(\beta-\tau) \hat{H}_{e e}} \hat{\Omega}^{\dagger} e^{-\tau \hat{H}_{e e}} \hat{\Omega}}{\operatorname{Tr}_{N} e^{-\beta \hat{H}_{e e}}} \equiv\left\langle\hat{\Omega}^{\dagger}(\tau) \hat{\Omega}(0)\right\rangle_{N}
$$

where $\hat{\Omega}^{\dagger}(\tau) \equiv \exp \left(\tau \hat{H}_{e e}\right) \hat{\Omega}^{\dagger} \exp \left(-\tau \hat{H}_{e e}\right)$ is the Euclidian-time Heisenberg operator. Inserting complete sets of $N$-body eigenstates of $\left.\hat{H}_{e e}(\{|i\rangle,|f|\rangle\}\right)$ with energies $E_{i, f}$ shows that 


$$
R_{\Omega}(\tau)=\frac{1}{Z} \sum_{i f} e^{-\beta E_{i}}|\langle f|\hat{\Omega}| i\rangle|^{2} e^{-\tau\left(E_{f}-E_{i}\right)},
$$

where $Z=\sum_{i} \exp \left(-\beta E_{i}\right)$ is the partition function. Thus, $R_{\Omega}(\tau)$ is the Laplace transform of the strength function $S_{\Omega}(E)$ :

$$
\begin{aligned}
& R_{\Omega}(\tau)=\int_{-\infty}^{\infty} e^{-\tau E} S_{\Omega}(E) d E \\
& S_{\Omega}(E)=\frac{1}{Z} \sum_{f i} e^{-\beta E_{i}}|\langle f|\hat{\Omega}| i\rangle|^{2} \delta\left(E-E_{f}+E_{i}\right) .
\end{aligned}
$$

It is important to note that we cannot usually obtain detailed spectroscopic information (i.e., energies and wave functions) from AFMC. Rather, we can calculate expectation values of operators in the thermodynamic ensembles or the ground state [1].

In the AFMC method, two-body interactions in $\hat{H}_{e e}$ are linearized through the Hubbard-Stratonovich (HS) transformation [7]. The difficult many-body evolution $U$ is replaced by a superposition of an infinity of tractable one-body evolutions, each in a different fluctuating external field, $\sigma$. Integration over the external fields thus reduces the many-body problem to quadrature, which is evaluated stochastically.

The many-body Hamiltonian can be written schematically as

$$
\hat{H}=\varepsilon \hat{\mathcal{O}}+\frac{1}{2} V \hat{\mathcal{O}} \hat{\mathcal{O}}
$$

where $\hat{\mathcal{O}}$ is a density operator of the form $a^{\dagger} a, V$ is the strength of the two-body interaction, and $\varepsilon$ a single-particle energy. In the full problem, there are many such quantities with various orbital indices that are summed over, but we omit them here for clarity.

All of the difficulty arises from the two-body interaction. If $\hat{H}$ were solely linear in $\hat{\mathcal{O}}$, we would have a one-body quantum system, which is readily dealt with. To linearize the evolution, we employ the Gaussian identity

$$
e^{-\beta \hat{H}}=\sqrt{\frac{\beta|V|}{2 \pi}} \int_{-\infty}^{\infty} d \sigma e^{-\frac{1}{2} \beta|V| \sigma^{2}} e^{-\beta \hat{h}} ; \hat{h}=\varepsilon \hat{\mathcal{O}}+s V \sigma \hat{\mathcal{O}} .
$$

Here, $\hat{h}$ is a one-body operator associated with a $c$-number field $\sigma$, and the many-body evolution is obtained by integrating the one-body evolution $\hat{U}_{\sigma} \equiv e^{-\beta \hat{h}}$ over all $\sigma$ with a Gaussian weight. The phase $s$ is 1 if $V<0$ or $i$ if $V>0$. Equation (8) is easily verified by completing the square in the exponent of the integrand and then doing the integral.

With an expression of the form (8), it is straightforward to write observables as the ratio of two integrals. For example, the canonical expectation value (2) becomes

$$
\langle\hat{\Omega}\rangle_{N}=\frac{\int d \sigma e^{-\frac{\beta}{2}|V| \sigma^{2}} \operatorname{Tr}_{N} \hat{U}_{\sigma} \hat{\Omega}}{\int d \sigma e^{-\frac{\beta}{2}|V| \sigma^{2}} \operatorname{Tr}_{N} \hat{U}_{\sigma}}=\frac{\int d \sigma W_{\sigma} \Omega_{\sigma}}{\int d \sigma W_{\sigma}},
$$

where $W_{\sigma}=G_{\sigma} \operatorname{Tr}_{N} \hat{U}_{\sigma}, G_{\sigma}=e^{-\frac{\beta}{2}|V| \sigma^{2}}$, and $\Omega_{\sigma}=\left(\operatorname{Tr}_{N} \hat{U}_{\sigma} \hat{\Omega}\right) /\left(\operatorname{Tr}_{N} \hat{U}_{\sigma}\right)$. Thus, the manybody observable is the weighted average (weight $W_{\sigma}$ ) of the observable $\Omega_{\sigma}$ calculated in a canonical ensemble involving only the one-body evolution $\hat{U}_{\sigma}$.

An expression of the form (9) has a number of attractive features. First, the problem has been reduced to quadrature. Second, all of the quantum mechanics is of the onebody variety, which scales simply with the square of the number of single-particle states 
included in the calculation. The price to pay is treating the one-body problem for all possible $\sigma$.

Since $\hat{H}_{e e}$ contains many two-body terms that do not commute, the evolution must be discretized, i.e., $\beta=N_{t} \Delta \beta$, before applying the HS transformation, i.e.,

$$
Z_{N}=\operatorname{Tr}_{N} e^{-\beta \hat{H}_{e e}} \rightarrow \operatorname{Tr}_{N}\left[e^{-\Delta \beta \hat{H}_{e e}}\right]^{N_{t}} \rightarrow \int \mathcal{D}[\sigma] G(\sigma) \operatorname{Tr}_{N} \prod_{n=1}^{N_{t}} e^{\Delta \beta \hat{h}\left(\sigma_{n}\right)}
$$

where $\sigma_{n}$ are the auxiliary fields at a given imaginary time-step $\Delta \beta, \mathcal{D}[\sigma]$ is the measure of the integrand, $G(\sigma)$ is a Gaussian in $\sigma$, and $\hat{h}$ is a one-body Hamiltonian. Dimensions of the integral can reach up to $10^{5}$ for systems of interest, and it is thus natural to use Metropolis random walk methods to sample the space. Because the numerical effort for AFMC scales only as a low power of the problem size, very large (and hence more realistic) calculations are possible.

The most significant challenge to applying the AFMC method resides in overcoming the fermion-sign problem. Algorithmic solutions to these problems are typically not robust and are highly dependent on the fermion system to which they are applied. For example, systems whose interactions are purely attractive are free of the sign problem [1].

For our system of many electrons confined in a quantum dot, the Coulomb forces are purely repulsive. In computing expectation values of observables as in Eq. (2), we will employ the finite-temperature methods discussed above. At high temperatures, these methods will not suffer from a Monte Carlo sign problem, but at lower temperatures, we will encounter the sign problem. Its most frequent manifestation occurs when the weight functions used for a Metropolis Monte Carlo evaluation of the integrals introduced by the Hubbard-Stratonovich transformation lose their positive-definite character [1]. We are exploring methods to stabilize the AFMC against the sign problem when the effective interactions include terms that are both attractive and repulsive $[9,10]$.

Previously, we developed the AFMC method for the nuclear shell-model problem and performed state-of-the-art calculations for a wide range of nuclear properties, including response functions at finite temperature $[1,11,12]$. The exciton density in the correlated nuclear system has been described by an application of AFMC techniques to the nuclei ${ }^{40} \mathrm{Ca}$ and ${ }^{42} \mathrm{Ca}$ in a very large model space [13].

\section{APPLICATION and RESULTS}

We consider the problem of $\mathrm{N}$ electrons of effective mass $m^{*}$ in a plane, $(x, y)$, confined by an external parabolic potential, $V(r)=\frac{1}{2} m^{*} \omega_{O}^{2} r^{2}$, and subject to a strong magnetic field $\vec{B}=B_{0} \vec{e}_{z}$. We consider the Zeeman splitting but neglect the spin-orbit interaction. The Hamiltonian for such a system is

$$
\hat{H}_{e e}=\sum_{i}\left[\frac{\left(\vec{p}-\frac{e}{c} \vec{A}\right)^{2}}{2 m^{*}}+V\left(r_{i}\right)+\frac{g^{*} \mu_{B}}{\hbar} \vec{B} \cdot \vec{S}_{i}\right]+\sum_{i<j} \frac{e^{2}}{\varepsilon\left|\vec{r}_{i}-\vec{r}_{j}\right|}
$$

where the vector potential is $\vec{A}\left(\vec{r}_{i}\right)=\left(B_{0} / 2\right)\left(-y_{i}, x_{i}, 0\right), g^{*}=0.54, \varepsilon=12.9, m^{*}=$ $0.067 m_{e}$, and $\hbar \omega_{0}=3 m e V$.

AFMC calculations require as basic inputs single-particle energies, two-body matrix elements of the interaction, and the initial many-body wavefunctions which we obtain 

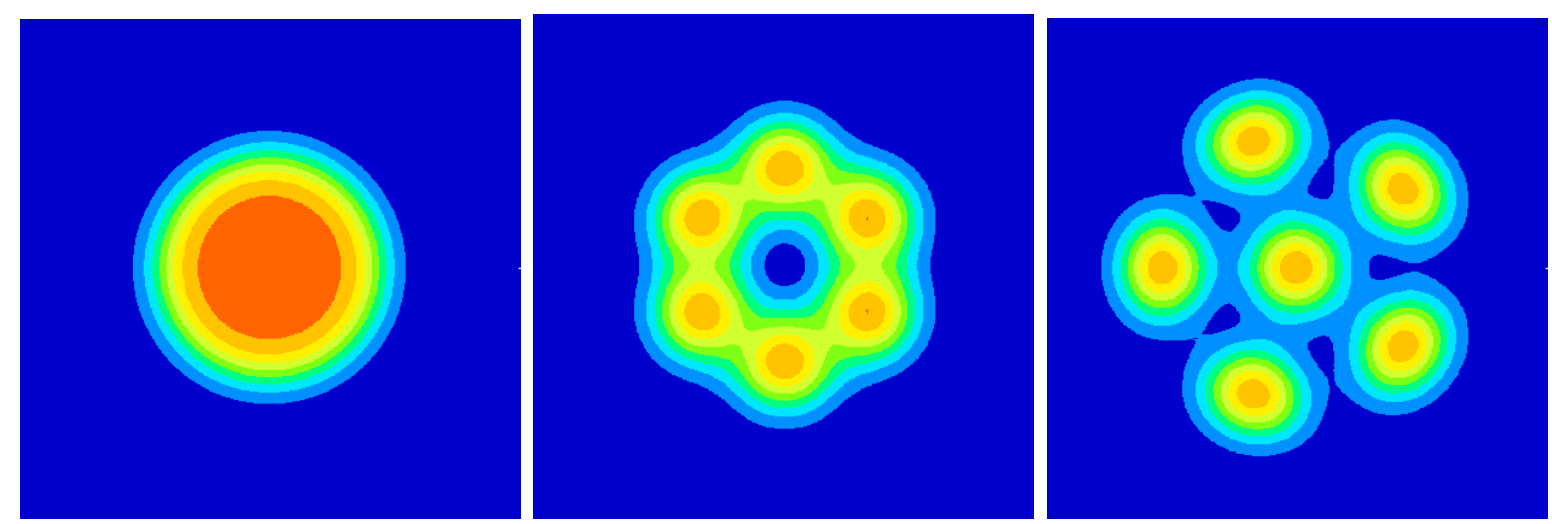

FIGURE 1. (a) Maximum-density droplet; (b) broken-symmetry phase, "Wigner molecule"; and (c) "Wigner crystal".

from Hartree-Fock (HF) calculations. The HF method provides physically meaningful single-particle states including the exchange interactions exactly [14].

Much experimental effort has focused on mapping the magnetic-field dependence of the structure of semiconductor quantum dots by measuring the chemical potential via capacitance spectroscopy [15]. Cusps and steps in the chemical potential were found to clearly separate different ranges of magnetic fields $[15,16]$. These features were identified with phase transitions in the charge density of the quantum dot. For increasing magnetic field, all electrons will become spin-polarized initiating the maximum density droplet (MDD) phase [15] [see Fig. (1a)]. In this phase, the density is constant and homogeneous at the maximum value that can be reached in the lowest Landau level. The stability of the MDD is determined by a competition between the kinetic and external confinement and the Coulomb repulsion between electrons. For increasing magnetic field, the chargedensity distribution of the droplet reconstructs [18] with a ring of electrons breaking off from the MDD phase (see Fig. (1b)). This edge reconstruction has been shown via mean-field calculations to result from a rotational symmetry-breaking phase transition to a Wigner molecule or Wigner crystal phase [14,17] [see Figs. [1b) and (1c)]. These calculations are in good qualitative agreement with recent experimental results [16].

While we are in the initial stages of testing and calibrating our AFMC code, we do have several interesting initial results. We begin with a canonical ensemble of $N=6$ electrons in a $B=4 \mathrm{~T}$ magnetic field. The corresponding Hartree-Fock solution is shown in Fig. (1a). We first demonstrate in Figure (2a) the behavior of the Monte Carlo sign $\langle\Phi\rangle$ as a function of inverse temperature $\beta$. Note that beyond approximately $\beta=80 \mathrm{eV}^{-1}$, the sign begins to drop quickly. In order to obtain good evaluations of expectation values beyond $\beta=100 \mathrm{eV}^{-1}$, we need to calculate many more Monte Carlo samples than here, where we have 2560 samples for each $\beta$. Figure $(2 \mathrm{~b})$ shows the occupation of the various Hartree-Fock states at different temperatures. Thermal excitation causes a degradation of the spin alignment, and thus an occupation of both spin-up and spin-down levels. An interesting near-term calculation will be to find the transition temperature when the return of spin polarization occurs.

We are at the beginning of an exciting and challenging study of the optical properties of quantum dots. At the present, we have a partially developed AFMC code with which to begin our work. We shall include computations of E1 dipole-transition operators in the code, and will investigate various techniques for overcoming the Monte Carlo sign problem for low-temperature studies. 

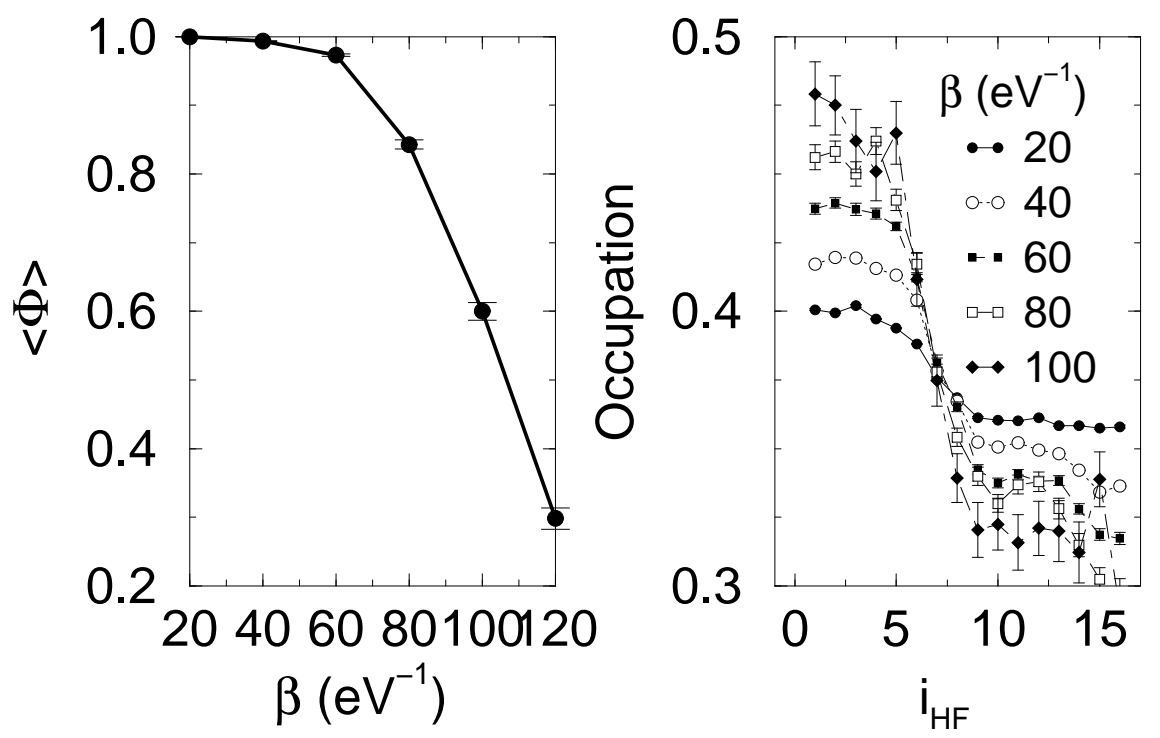

FIGURE 2. Left: a) sign problem as a function of inverse temperature; right: b) occupation of the HF states, $i_{H F}$ with decreasing temperature.

\section{ACKNOWLEDGEMENTS}

Research sponsored by the Laboratory Directed Research and Development Program of Oak Ridge National Laboratory, managed by Lockheed Martin Energy Research Corp. for the U. S. Department of Energy under Contract No. DE-AC05-96OR22464.

\section{REFERENCES}

1. S.E. Koonin, D.J. Dean, and K. Langanke, Phys. Rep. 278, 1 (1997).

2. L. Jacak, P. Hawrylak, and A. Wójs, Quantum Dots, (Springer, Berlin, 1997).

3. L. Landin, et al., Science 280, 262 (1998).

4. G.J. Iafrate and M.A. Stroscio, IEEE Trans. Electron Devices 43, 1621 (1996).

5. K. Hess, Challenges in Nanostructure Simulation, ITRI Workshop on Vision for Nanotechnology R \& D in the Next Decade, January 1999, Arlington, VA.

6. G. Sugiyama and S.E. Koonin, Ann. Phys. 168, 1 (1986).

7. J. Hubbard, Phys. Rev. Lett. 3, 77 (1959); R.L. Stratonovich, Dokl. Akad. Nauk. S.S.S.R. 115, 1097 (1957).

8. W. von der Linden, Phys. Rep. 220, 53 (1992).

9. N. Rom, et al., Chem. Phys. Lett. 270, 382 (1997).

10. Shiwei Zhang, J. Carlson, and J.E. Gubernatis, Phys. Rev. B 55, 7464 (1997).

11. D.J. Dean, et al., Phys. Rev. C 58, 536 (1998).

12. P.B. Radha, et al., Phys. Rev. C 56, 3079 (1997).

13. D.J. Dean and S.E. Koonin, Phys. Rev. C 60, 54306 (1999).

14. H.-M. Müller and S.E. Koonin, Phys. Rev. B 54, 14532 (1996).

15. R. C. Ashoori, Nature 379, 413 (1996).

16. T.H. Oosterkamp, et al., Phys. Rev. Lett. 82, 2931 (1999).

17. S.M. Reimann, et al., Phys. Rev. Lett. 83, 3270 (1999).

18. C. de C. Chamon and X.G. Wen, Phys. Rev. B 49, 8227 (1994). 